\title{
Microstructure electrodes as a means of creating uniform discharges at atmospheric pressure
}

\author{
K.-H. Gericke*, C. Geßner, P. Scheffler \\ Institut für Physikalische Chemie, Technische Universität Carolo Wilhelmina zu Braunschweig, Hans-Sommer-Str.10, \\ D-38106 Braunschweig, Germany
}

\begin{abstract}
Large-area direct current (DC) discharges were generated using planar microelectrode arrays (MEAs) with electrode distances between 50 and $250 \mu \mathrm{m}$. A homogenous diffuse light emission with a typical thickness of approximately $0.5-$ $1.5 \mathrm{~mm}$ was observed. The discharge can be operated over a wide pressure range with sustaining voltages of only a few $100 \mathrm{~V}$ DC and showed the characteristics of a normal glow discharge. Possible areas of application of the MEA were surface modification of materials, plasma chemistry, pollution control and as light sources. The discharge was characterized via optical emission spectroscopy (OES). The temperature of excited species in the discharge was derived from $\mathrm{OH}$ emission spectra. A temperature of about $3000 \mathrm{~K}$ was calculated. Laser-induced fluorescence (LIF) spectra of NO $\left(\mathrm{A}^{2} \Sigma \leftarrow \mathrm{X}^{2} \Pi_{1 / 2}\right)$ in the ground state indicated that the gas temperature of the discharge was only a few ten degrees above room temperature. (C) 2002 Elsevier Science Ltd. All rights reserved.
\end{abstract}

Keywords: Plasma sources; Microelectrode; Non-thermal gas discharge; OES; LIF

\section{Introduction}

Non-thermal plasma processing techniques have become essential tools in industrial processing and manufacturing. They are well established in a wide range of applications including pollution control, surface modification of materials or lighting technology. The applied weakly ionized plasmas are highly non-equilibrium, i.e. the electrons have large mean kinetic energies equivalent to several $10000 \mathrm{~K}$, whereas the gas remains at ambient temperature. The technically most important method to generate a non-thermal plasma is by high electric field (electrical discharge). For that,

\footnotetext{
*Corresponding author. Fax: +49-531-391-5396.

E-mail address: k.gericke@tu-bs.de (K.-H. Gericke).
}

direct current (DC), alternating current (AC), or radio frequency $(\mathrm{RF})$ up to the gigahertz range can be applied. Since the basic electrical discharge creation process in gases proceeds via electron impact ionization, electrons must be accelerated by the applied field $E$ over the mean free path $\lambda$ to gain a minimum kinetic energy

$E_{k i n}=e E \lambda>E_{e x}$,

where $E_{e x}$ is the threshold energy necessary for electronic excitation, dissociation, fragmentation or ionization of the gas. Therefore, operation of electrical discharge devices requires either a low gas pressure, i.e. large $\lambda$ or a very strong electric field $E=U / d$. Under these circumstances, the application of plasma technology is often quite 
complex and expensive. Therefore, it is sought to facilitate the application of plasma sources and to minimize their cost. To this aid, it is desirable to achieve stable and reliable operation of plasma sources at or only slightly below atmospheric pressure, employing moderate voltages in the range of several hundred volts.

One promising approach for a discharge device, which operates without any vacuum restraints and at moderate voltages, are electrode arrays fabricated by means of micromachining technologies, which can provide distances on a micron scale between the electrode elements. Here, even at moderate voltages, the electrostatic field can exceed the threshold value required to initiate the electrical breakdown process. The breakdown voltage $V_{b}$ of a gas discharge and the corresponding breakdown field strength depend on the gas, the material of the cathode, the pressure and the discharge gap width. This is expressed in the $p d$ law and the corresponding Paschen curves $V_{b}(p d)$. In order to generate gas discharges at pressures up to $1 \mathrm{~atm}$, applying only moderate voltages, the discharge gap width has to be optimized to operate the discharge in the minimum of the corresponding Paschen curve. The field between the microelectrodes easily exceeds $10^{5} \mathrm{~V} / \mathrm{cm}$ and can approach $10^{6} \mathrm{~V} / \mathrm{cm}$ and even higher values at the edges of the electrodes. For example, a DC voltage of $U=1000 \mathrm{~V}$ applied to a microelectrode array (MEA) with an electrode gap of $d=20 \mu \mathrm{m}$ results in an electric field $E$ of $5 \times 10^{7} \mathrm{~V} / \mathrm{m}$. In this regard, it is possible to produce gas discharges up to atmospheric pressure by means of MEA with very moderate voltages so that a wide area of plasma applications becomes feasible under reasonable conditions.

\section{Experimental procedure}

\subsection{Planar microelectrode arrays}

Fig. 1 shows a part of a layout and explains the basic geometry of the MEA we have studied. The planar MEA consists of parallel metal strips with a length of about $3 \mathrm{~cm}$, which are bonded to a substrate and form, alterna-

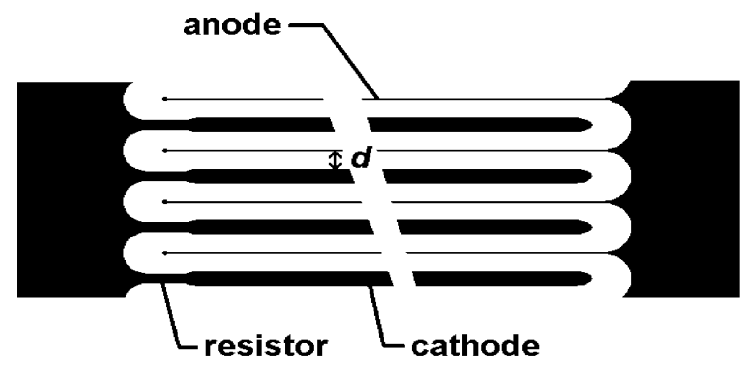

Fig. 1. Scheme of the planar microelectrode arrays.

tively, anodes and cathodes. Between 40 and 270 strips with elliptically formed ends and a width ranging from 50 to $400 \mu \mathrm{m}$ act as cathodes. An area with a width of about $4 \mathrm{~mm}$ and a length of $40 \mathrm{~mm}$, where the electrical contact with the mounting is established, links together all cathodes, so that a comb-like structure originates. A similar structure is formed by the anodes, which are 12 or $25 \mu \mathrm{m}$ wide, respectively. The anodes end with a circle, which is $50 \mu \mathrm{m}$ in diameter. The electrode gap $d$ was varied from 50 to $250 \mu \mathrm{m}$.

At low pressures up to about 30 Torr $(4000 \mathrm{~Pa})$ it is possible to generate large-area homogeneous discharges with a low power consumption of the arrays. Therefore, the voltage drop is relatively small when breakdown occurs and the electrodes can be regarded as fully decoupled. However, this behavior changes, when the pressure is increased. As a consequence, breakdown often occurs merely in a limited area of the array. Therefore, it is necessary to develop an array design which allows a creation of a large-area discharge as a combination of a great number of microdischarges decoupled with reference to their power consumption. The decoupling of the electrodes is achieved by adding an individual resistor to each cathode.

The MEA discussed in this paper were standard photomasks. Using e-beam technology, the arrays were made of glass substrates on which a chromium layer with a thickness of $0.1 \mu \mathrm{m}$ was evaporated. On a $4 \times 4 \mathrm{in}^{2}$ substrate, four arrays could be manufactured, yielding a total array size of about $5 \times 5 \mathrm{~cm}^{2}$. 


\subsection{Operation and spectroscopic measurements}

Fig. 2 schematically shows the experimental setup for the studies of the MEA as an electrical discharge device. All measurements were performed in a cubical chamber made of stainless steel with a total volume of about 1.51. For all experiments, except for the quantitative laserinduced fluorescence (LIF) measurements, which were performed under static conditions, a gas-flow rate between 10 and $50 \mathrm{sccm}$ was set up by means of mass flow controller. Experiments were carried out in the following gases: helium, neon, argon, krypton, xenon and nitrogen. Besides, experiments with water vapor were performed. For the operation with DC, a high voltage supply was used, which could provide voltages up to $5 \mathrm{kV}$. A ballast resistor was employed in series to limit the discharge current. For determining the currentvoltage characteristics, i.e. the sustaining voltage of the discharge, a digital multimeter in parallel connection was used. The current value was provided by the power supply. The voltages applied in order to generate the discharge ranged from -1500 to $-2500 \mathrm{~V}$. The sustaining voltage of the discharge $U_{S}$ was in the range from -200 to $-500 \mathrm{~V}$ depending on the array, the gas and the gas pressure. The discharge current was between 0.1 and $2.0 \mathrm{~mA}$. The current was limited to $2 \mathrm{~mA}$, in order to avoid damage of the arrays at high currents due to heating.

For optical emission spectroscopy (OES) measurements, the light emitted by the excited species is focused onto the entrance slit of a CzernyTurner monochromator (1200 g/mm grating, focus $=500 \mathrm{~mm}$ and resolution $=0.03 \mathrm{~nm}$ ) and detected by a photomultiplier tube. The photomultiplier tube output is amplified and registered by a personal computer as a function of the monochromator setting, yielding the OES spectrum. For temperature measurements, the experimental setup was outlined for the detection of nitrogen oxide (NO) via LIF technique (see Fig. 2). Tunable laser light with a wavelength around $226 \mathrm{~nm}$ probed the discharge volume a few millimeters above the MEA surface. The ultraviolet laser radiation is obtained by second-harmonic generation of the radiation emitted by a tunable pulsed dye laser operated at Coumarin 47 and pumped by a $\mathrm{XeCl}$ excimer laser. The laser beam is

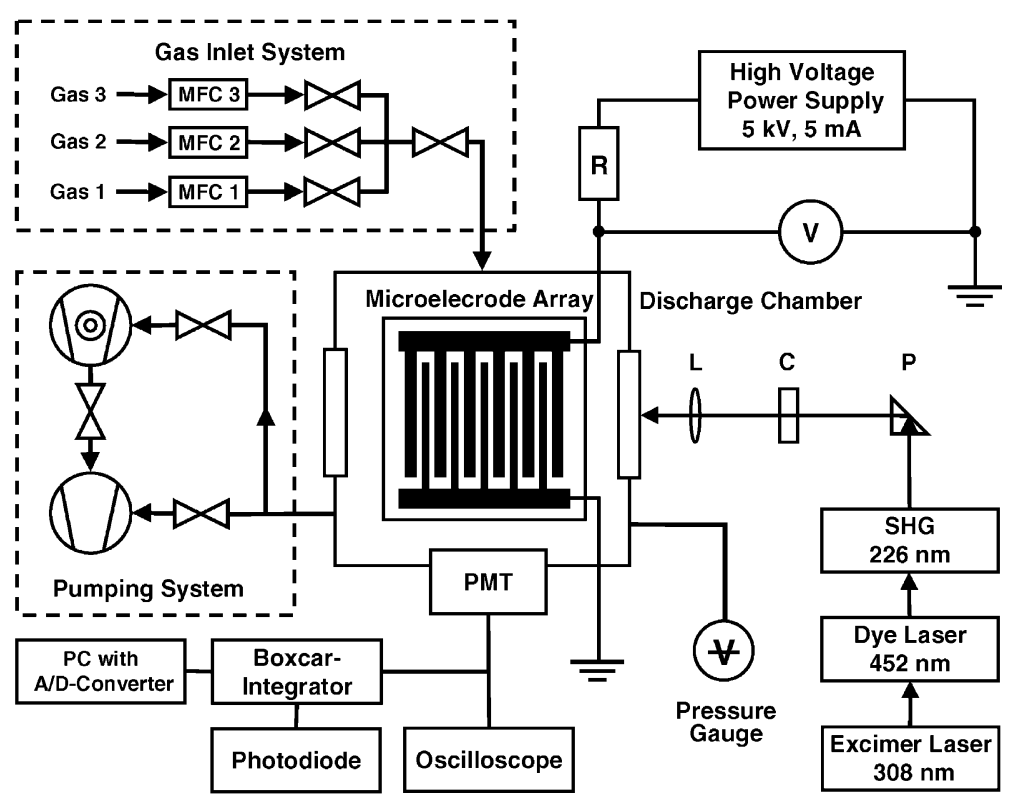

Fig. 2. Scheme of the experimental setup. 
expanded using a combination of cylindrical and converging lenses to form a laser sheet. The emitted fluorescence light was detected under $90^{\circ}$ by a photomultiplier. The multiplier signal was monitored by means of a digital oscilloscope and was submitted to a gated integrator and boxcar averager, which was triggered by a photodiode. Finally, the signal was transferred to a personal computer with an A/D-converter.

\section{Results and discussion}

\subsection{Working range and general optical appearance}

A uniform discharge covering the whole active area of the array $\left(4 \times 3 \mathrm{~cm}^{2}\right)$ could be generated up to 40 Torr $(5300 \mathrm{~Pa})$ in helium and neon. In argon, krypton, xenon, nitrogen and water vapor discharges are stable up to 5-10 Torr $(670-1330 \mathrm{~Pa})$. When the pressure is increased beyond the maximum values mentioned above, the area covered by the discharge decreases continuously with increasing pressure in all gases. The maximum pressure at which a discharge can be generated with the MEA under investigation is about 100 Torr $(13300 \mathrm{~Pa})$ in helium and neon and about 40 Torr $(5300 \mathrm{~Pa})$ in the other gases. For all gases, the light emission is observed from a region just above the MEA with a typical thickness of approximately $0.5-1.5 \mathrm{~mm}$.

The discharge showed the electrical characteristics of a normal glow discharge. The currentvoltage characteristic has a positive slope, i.e. the discharge shows a resistive behavior in the studied pressure range. A simple estimate of the power consumption yields a value between 0.015 and $0.05 \mathrm{~W} / \mathrm{cm}^{2}$.

\subsection{Optical characterization}

\subsubsection{OES measurements}

Fig. 3 shows a helium spectrum at a wavelength range from 350 to $750 \mathrm{~nm}$, monitored in a helium gas discharge at 3.6 Torr $(480 \mathrm{~Pa})$ at a constant helium flow of about $10-20 \mathrm{sccm}$. All observed transitions can be assigned to excited states of the helium atom, as indicated in the spectrum, no lines

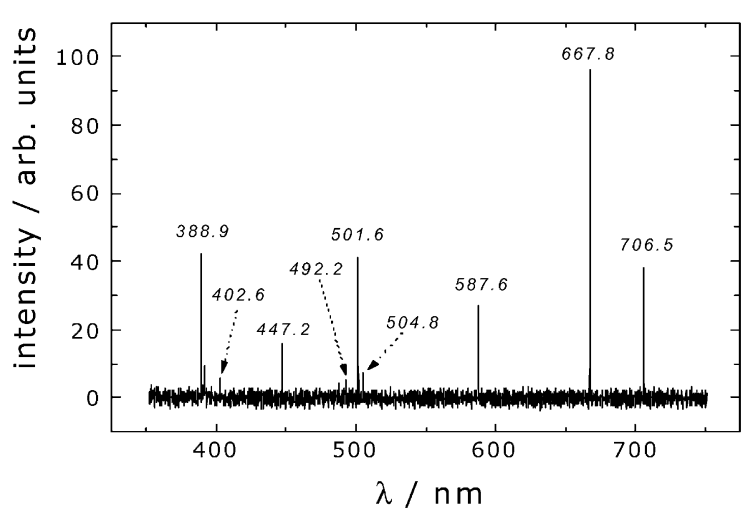

Fig. 3. Helium emission spectrum.

originating from $\mathrm{He}^{+}$ions were found. In a neon gas discharge at 2 Torr $(270 \mathrm{~Pa})$, the transitions $\left(2 p^{5} 3 s^{1}-2 p^{5} 3 p^{1}\right)$ of the neutral neon atom is observed. As in the case of helium, no lines can be observed which originate from neon ions.

The spectra of the succeeding noble gases differ from the helium and neon spectra in a way that, in addition to the atomic transitions between highly excited electronical states $\left(n \mathrm{p}^{5}[n+1] \mathrm{s}^{1}-\right.$ $n \mathrm{p}^{5}[n+1] \mathrm{p}^{1}, n=3,4,5$ for $\mathrm{Ar}, \mathrm{Kr}$ and $\mathrm{Xe}$ ), also transitions of the singly positive ions $\mathrm{Ar}^{+}, \mathrm{Kr}^{+}$ and $\mathrm{Xe}^{+}$are detected. Spectra monitored in a $\mathrm{N}_{2}$ gas discharge at a pressure of about 1.5 Torr $(200 \mathrm{~Pa})$ indicate transitions in the wavelength range from 296.2 to $434.4 \mathrm{~nm}$ which mostly belong to the second positive system $\left(\mathrm{C}^{3} \Pi_{\mathrm{u}} \rightarrow \mathrm{B}^{3} \Pi_{\mathrm{g}}\right)$ of the $\mathrm{N}_{2}$ molecule. In addition, ionic lines which can be assigned to the first negative system $\left(\mathrm{B}^{2} \Sigma_{\mathrm{u}}^{+} \rightarrow \mathrm{X}^{2} \Sigma_{\mathrm{g}}^{+}\right)$ of $\mathrm{N}_{2}^{+}$are detected.

$\mathrm{OH}$ emission spectra were taken in a pure $\mathrm{H}_{2} \mathrm{O}$ vapor atmosphere at low pressures of about 0.4 Torr $(53 \mathrm{~Pa})$. The monitored spectrum presented in Fig. 4 indicates two $\mathrm{OH}$ band heads at 281.1 and $306.4 \mathrm{~nm}$ out of the system $\mathrm{A}^{2} \Sigma \rightarrow \mathrm{X}^{2} \Pi$. The rotational structure is not resolved.

The emission spectra are used to estimate the magnitude of energy delivered by the gas discharge generated using MEA in various gases, in particular, the available energy of the electrons to excite, ionize or fragment the neutral species. The highest excited state of helium atoms detected is the $5^{3} \mathrm{D}_{3,2,1}$ state, followed by $4^{1} \mathrm{D}_{2}$ and $4^{1} \mathrm{~S}_{0}$. These states possess energies between 23.5 and 


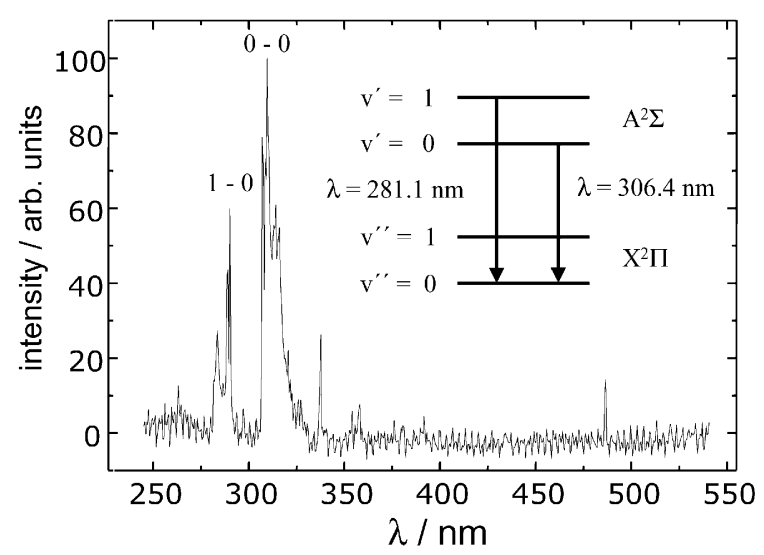

Fig. 4. $\mathrm{OH}$ emission spectrum.

$24 \mathrm{eV}$. To populate these highly excited states, sequential excitation is possible via the lowest excited helium states $2^{1} \mathrm{~S}_{0}$ and $2^{3} \mathrm{~S}_{1}$ with state energies of 20.6 and $19.8 \mathrm{eV}$. Therefore, at least some electrons possess energies up to $20.6 \mathrm{eV}$, causing excitation from the ground state $1^{1} \mathrm{~S}_{0}$ to the $2^{1} \mathrm{~S}_{0}$ and $2^{3} \mathrm{~S}_{1}$ states. This is not an average electron energy, but represents the high-energy tale of the energy distribution. The most intensive transition observed in the helium spectrum is the $3^{1} \mathrm{D}_{2}-2 \mathrm{P}_{1}$ transition.

Argon, krypton and xenon emission spectra indicate the presence of singly positive rare gas ions, whereas helium and neon spectra only show transitions of highly excited rare gas atoms. Considering the ionization energies of the noble gases, this observation can be rationalized: Only argon, krypton and xenon exhibit ionization energies lower than $20.6 \mathrm{eV}$, the expected electron energy according to the emission spectra of helium. The number densities of electrons above $20.6 \mathrm{eV}$ are low, and thus, ionization of neon or helium with their high ionization energies of 21.6 or $24.6 \mathrm{eV}$, respectively, becomes unlikely.

Apart from estimations of the electron energies, the temperature of the other species in the gas discharge, atoms, ions, radicals or molecules is of interest. At a first glance, it seems to be reasonable to extract this temperature from the $\mathrm{OH}$ emission spectrum shown in Fig. 4. Following the Boltzmann law (2), the number of molecules in a state $N$ at a temperature $T$ depends on the state energy $E$.
Since the lowest energy which is the energy of the vibronic ground state $E_{0}$ can be set to zero, the equation can be written as follows:

$T=\frac{E_{1}}{k} \frac{1}{\ln N_{0} / N_{1}}$.

$E_{1}$, the energy of the vibrational state $v^{\prime}=1$ in the electronic excited state of $\mathrm{OH}\left({ }^{2} \Sigma\right)$ can be extracted from spectroscopic data. The fluorescence intensities $I$ for the transition from the vibrational state $v$ of the electronic excited state to the ground state $\mathrm{A}^{2} \Sigma \rightarrow \mathrm{X}^{2} \Pi$ are proportional to the product of the transition probabilities $A_{v^{\prime} v^{\prime \prime}}$ and the number of molecules $N_{v}$ in a state $v$. The intensities of both transitions $v^{\prime}=1 \rightarrow v^{\prime \prime}=0$ and $v^{\prime}=0 \rightarrow v^{\prime \prime}=0$ are obtained from integration over the rotational structures of both bands. The transition probabilities $A_{v^{\prime} v^{\prime \prime}}$ are well known, and thus, Eq. (2) can be used to obtain the temperature $T$, provided the proportional constants do not change for both transitions: $T=(3370 \pm 250) \mathrm{K}$. This temperature is far too high to represent the gas temperature in the plasma, because the Boltzmann law demands a statistical distribution of species between all states, which is not reached during the lifetime of the $\mathrm{A}^{2} \Sigma$ state $(\tau=693 \mathrm{~ns})$.

\subsubsection{LIF measurements}

To determine the actual gas temperature, analysis of the population of the rotational states of LIF spectra is more suitable, because this technique allows the characterization of molecules in their electronic ground state under equilibrium conditions. Therefore, the LIF spectra of the $\mathrm{A}^{2} \Sigma^{+} \rightarrow \mathrm{X}^{2} \Pi_{1 / 2}$ transition of nitrogen oxide (NO) are measured by scanning the laser excitation wavelength from 225.5 to $226.4 \mathrm{~nm}$. Fig. 5 presents a NO LIF spectrum, measured in a gas mixture of $500 \mathrm{ppm} \mathrm{NO}$ in helium at a total pressure of 11.4 Torr $(1520 \mathrm{~Pa})$. All observed lines can be assigned to the rotational transitions belonging to the $\mathrm{R}_{1}, \mathrm{R}_{21}$ and $\mathrm{Q}_{1}$ branches of the $v^{\prime}=0 \rightarrow v^{\prime \prime}=0$ band of the $\mathrm{A}^{2} \Sigma^{+} \rightarrow \mathrm{X}^{2} \Pi_{1 / 2}$ electronic transition. The rotational lines of the $R_{1}$ branch are well resolved and are used to determine the gas temperature $T$ according to

$I \sim S_{J^{\prime} J^{\prime \prime}}\left(2 J^{\prime \prime}+1\right) \exp \left(-E_{r o t} / k T\right)$, 


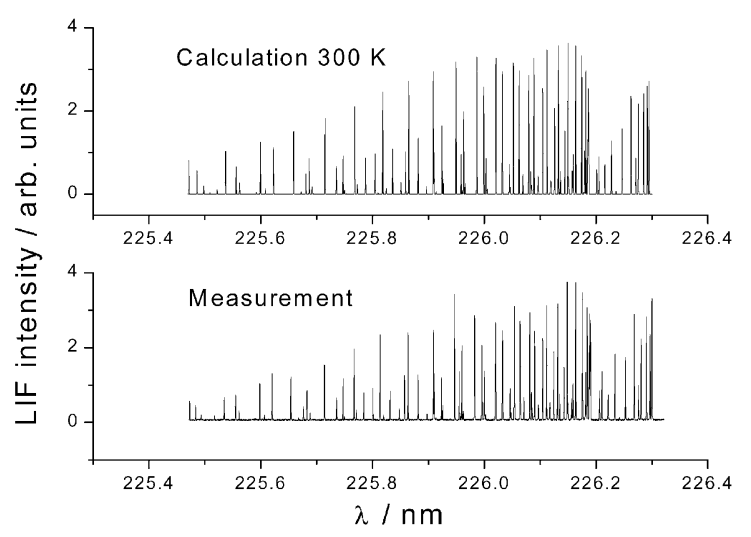

Fig. 5. (Top) Calculated spectrum, $T=300 \mathrm{~K}$. (Bottom) NO LIF spectrum of the $v^{\prime}=0 \leftarrow v^{\prime \prime}=0$ band of the $\mathrm{A}^{2} \Sigma^{+} \leftarrow \mathrm{X}^{2} \Pi_{1 / 2}$ transition.

where $I$ is the intensity of lines belonging to the $\mathrm{R}_{1}$ branch and $E_{\text {rot }}$ the rotational energy of the lower $J^{\prime \prime}$ state. Plotting $\ln \left[I /\left(2 J^{\prime \prime}+1\right)\right]$ versus the rotational energy $E_{r o t}$, as presented in Fig. 6, the temperature $T$ can be determined from the slope of the resulting line. The temperature obtained without plasma $T=289 \pm 14 \mathrm{~K}$ corresponds to room temperature, the temperature with the ignited $T=316 \pm 24 \mathrm{~K}$ is only a few ten degrees higher. Considering the margins of error being of the same order as the difference in temperature, the influence of plasma ignition on the temperature is insignificant. The Boltzmann plot for the $R_{1}$ branch of the $(0-0)$ band of the $\mathrm{A}^{2} \Sigma^{+} \leftarrow \mathrm{X}^{2} \Pi_{1 / 2}$ transition in the NO molecule is given in Fig. 6. The temperature is determined from the slope $(-1 / k T)$. For comparison, Fig. 5 shows not only the measured NO spectrum, but the spectrum calculated for $T=300 \mathrm{~K}$, as well. The temperatures $T \approx 300 \mathrm{~K}$ obtained from the Boltzmann plot, as stated above, are in good agreement with the temperature of the calculated spectrum. Thus, the MEA discharge device provides a non-equilibrium plasma: this is a two-temperature system, containing "hot" electrons with kinetic energies of up to $20 \mathrm{eV}$ inducing excitation, fragmentation or ionization as well as atoms, molecules, ions and radicals, with kinetic energies equivalent to room temperature.

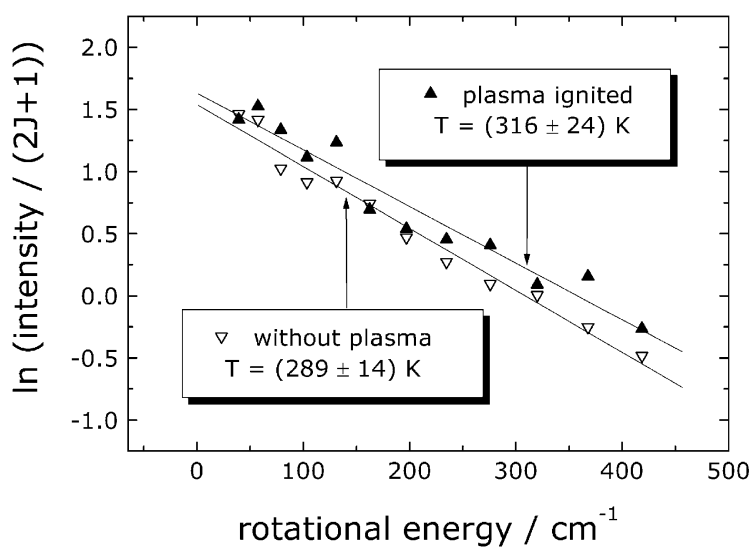

Fig. 6. The Boltzmann plot for the $\mathrm{R}_{1}$ branch of the $(0-0)$ band of the $\mathrm{A}^{2} \Sigma^{+} \leftarrow \mathrm{X}^{2} \Pi_{1 / 2}$ transition in the NO molecule.

\section{Conclusion}

Discharges generated using MEA provided a two-temperature system, containing hot electrons with energies up to $20 \mathrm{eV}$ and molecules, ions or atoms with temperatures of about $320 \mathrm{~K}$. The high-energy electrons provided sufficient energy to ionize or fragment molecules, without raising the gas temperature of the discharge significantly above room temperature. The main advantage of this low-temperature plasma is that a variety of plasma-chemical reactions (e.g. plasma-enhanced chemical synthesis, surface treatment, exhaust gas cleaning processes) can be carried out in a rather cold gas phase. Furthermore, because of their microscaled dimensions, MEA are ideally applicable as devices in microreactors.

The results reported are the first part of a comprehensive study on planar MEAs. They confirmed the expectation that the arrays could act as an efficient discharge device in a wide range of applications in the plasma technology. Detailed investigations are in progress [1-4], demonstrating the potential of MEA to generate large-area discharges at atmospheric pressure. Recent investigations show, that MEA operated at RFs $(13.56 \mathrm{MHz})$ at the power densities $20-50 \mathrm{~W} / \mathrm{cm}^{2}$ provide atmospheric pressure discharges in helium, argon, nitrogen and in various gas mixtures. 
These atmospheric pressure discharges have been used to decompose various exhaust gases, for example $\mathrm{NO}_{x}$ or $\mathrm{CF}_{4}$.

MEA are also feasible as a novel kind of catalyst for plasma-chemical processes, providing high electron energies in a rather cold gas phase. A variety of plasma-enhanced syntheses can be carried out, for example, the production of acetylene and higher hydrocarbons out of methane. Furthermore, the deposition of thin carbon films, using discharges in mixtures of methane or acetylene in helium is under investigation.

\section{Acknowledgements}

This work was supported by the "Deutsche Bundesstiftung Umwelt" (DBU), and the "Bun- desministerium für Bildung und Forschung" (BMBF), Germany.

\section{References}

[1] Scheffler P, Geßner C, Gericke K-H. Contributed paper to the 7th International Symposium on High Pressure Low Temperature Plasma Chemistry, HAKONE VII, September 10-13, 2000, Greifswald. p. 407-11.

[2] Geßner C, Scheffler P, Gericke K-H. Contributed paper to the 7th International Symposium on High Pressure Low Temperature Plasma Chemistry, HAKONE VII, September 10-13, 2000, Greifswald. p. 112-6.

[3] Geßner C, Scheffler P, Gericke K-H. Proceedings of the XIII International Conference on Gas Discharges and their Applications, GD 2000, September 3-8, 2000, Glasgow. p. $548-51$.

[4] Geßner C, Scheffler P, Gericke K-H. Proceedings of the XXV International Conference on Phenomena in Ionized Gases, XXV ICPIG, vol. 4, July 17-22, 2001, Nagoya. p. $151-2$. 